\title{
Florística e fitossociologia de uma floresta de vertente na Amazônia Central, Amazonas, Brasil ${ }^{1}$.
}

\author{
Arlem Nascimento de OLIVEIRA², Iêda Leão do AMARAL ${ }^{3}$
}

\begin{abstract}
RESUMO
O estudo florístico e fitossociológico de árvores, palmeiras e lianas com diâmetro à altura do peito (DAP) $\geqslant 10 \mathrm{~cm}$, em uma

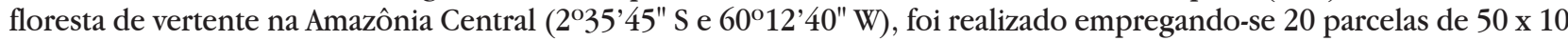
$\mathrm{m}$, distribuídas em dois transectos paralelos de $500 \times 10 \mathrm{~m}$. Foram registrados 771 indivíduos, pertencentes a 50 famílias, 120 gêneros e 239 espécies. Das espécies amostradas, 44\% são "localmente raras". Sapotaceae, Lecythidaceae, Fabaceae, Caesalpiniaceae e Chrysobalanaceae constituíram as cinco famílias com maior riqueza de espécies e número de indivíduos. Dos 771 indivíduos amostrados, mais de 65\% apresentaram DAP $\geqslant 20 \mathrm{~cm}$. As espécies Eschweilera bracteosa e Protium apiculatum apresentaram os maiores valores de IVI. Cerca de 83\% das espécies encontram-se distribuídas aleatoriamente no hectare amostrado. O índice de diversidade Shannon-Wiener foi de 5,01 nats.indivíduo ${ }^{-1}$, com uniformidade de 0,91 , valores altos no contexto de levantamentos semelhantes na região. A heterogeneidade edáfica e topográfica da área, as taxas de recrutamento de novos indivíduos e de espécies "localmente raras" à comunidade local, podem ter contribuído para as altas dissimilaridade $(36,2 \%)$ e diversidade florísticas documentadas neste estudo.
\end{abstract}

PALAVRAS-CHAVE

fitossociologia, diversidade, Terra firme, Amazônia Central

\section{Floristic and phytosociology of a slope forest in Central Amazonia, Amazonas, Brazil.}

\begin{abstract}
The floristic and phytosociological study of trees, palms and lianas with diameter at breast height (DBH) $\geqslant 10 \mathrm{~cm}$ in a

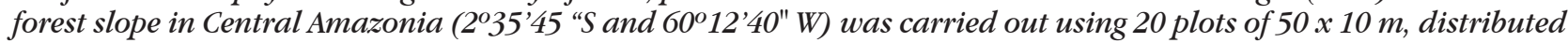
in two parallel transects of $500 \times 10 \mathrm{~m}$. A total of 771 plants were registered, belonging to 50 families, 120 genera and 239 species. Of the sampled species, $44 \%$ are locally rare. Families with the most species and number of individuals were Sapotaceae, Lecythidaceae, Fabaceae, Caesalpiniaceae and Chrysobalanaceae. More than 65\% of the sampled plants had DBH $\geqslant 20 \mathrm{~cm}$. Eschweilera bracteosa and Protium apiculatum were the most important in terms of IVI. Almost 83\% of the species were randomly distributed in the sampled hectare. The Shannon-Wiener diversity index (5.01 nats. individual ${ }^{-1}$ ) and eveness (0.91) were high when compared to similar surveys in the same region. The edaphic and topographic heterogeneity, recruitment rates of new individuals and locally rare species at the community level, may have contributed to the high dissimilarity (36.2\%) and floristic diversity registered in this study.
\end{abstract}

\section{KEY WORDS}

phytosociology, diversity, upland, Central Amazonia

\section{INTRODUÇÃO}

A região amazônica ocupa aproximadamente $6.000 .000 \mathrm{~km}^{2}$ da América do Sul, sendo constituída por diferentes tipos de vegetação. Cerca de $65 \%$ dessa região é coberta por um tipo florestal denominado floresta de terra firme, caracterizada principalmente pela elevada riqueza e diversidade de espécies (Prance, 1976; Amaral,
1996; Oliveira \& Mori, 1999; Lima Filho et al., 2001; Oliveira et al., 2003).

A Amazônia há muito vem despertando interesse tanto nacional como internacional. Grande parte desse interesse deve-se não apenas à sua extensão territorial e/ou potencial econômico, mas sobretudo, às questões relacionadas ao crescente desflorestamento da região nos últimos anos (Laurance et al., 2001). A destruição de extensas coberturas

\footnotetext{
${ }^{1}$ Trabalho executado com auxílio financeiro do CNPq, PPD-G7/LBA.

${ }^{2}$ Estudante de Pós-Graduação da Universidade Federal do Amazonas - UFAM. Manaus, AM. Email: arlem@inpa.gov.br

${ }^{3}$ Instituto Nacional de Pesquisas da Amazônia - INPA/CPBO. Manaus, AM. Email: iamaral@inpa.gov.br
} 


\section{ACTA \\ AMAZONICA}

FLORÍSTICA E FITOSSOCIOLOGIA DE UMA FLORESTA

DE VERTENTE NA AMAZÔNIA CENTRAL, AMAZONAS, BRASIL vegetais, além de provocar perdas imensuráveis de recursos genéticos, pode contribuir de maneira decisiva nas mudanças climáticas regionais e globais (Nobre et al., 1991; Skole \& Tucker, 1993; Houghton et al., 2000).

A floresta amazônica é o maior reservatório natural da diversidade vegetal do planeta, onde cada um de seus diferentes ambientes florestais possui um contigente florístico rico e variado, muitas vezes exclusivo de determinado ambiente. As múltiplas inter-relações entre seus componentes bióticos e abióticos, formam um conjunto de ecossistemas altamente complexo e de equilíbrio ecológico extremamente frágil.

Os estudos estruturais e florísticos desenvolvidos na Amazônia têm demonstrado que os ambientes florestais de terra firme, ou seja, as florestas de platô e vertente, apresentam alta diversidade, representada por poucos indivíduos de cada espécie (Porto et al., 1976; Prance et al. 1976; Amaral, 1996; Oliveira, 1997; Lima Filho, 2001) e alta dissimilaridade florística entre parcelas adjacentes (Campbell et al., 1986; Ferreira \& Prance, 1998).

A comunidade vegetal de vertente representa uma faixa de transição entre as florestas de platô e campinarana (Ribeiro et al., 1999). Nas partes mais altas é floristicamente semelhante à comunidade de platô (Tello, 1995) e, nas mais baixas, fisionomicamente parecida com a campinarana (Ribeiro et al., 1999), porém sem apresentar as espécies que a caracteriza. Por outro lado, outras espécies são exclusivas desse ecossistema florestal (Tello, 1995; Ribeiro et al., 1999).

Fatores edafo-climáticos como solos relativamente mais férteis em nutrientes e a alta precipitação sem uma sazonalidade marcante, têm sido citados como possíveis responsáveis pela alta diversidade de plantas na Amazônia (Gentry, 1988; Clinebell et al., 1995; Steege et al., 2000). Outros autores relacionam maior riqueza de espécies à dinâmica natural de mortalidade de árvores, onde florestas com altas taxas de mortalidade e recrutamento seriam mais diversificadas (Phillips et al., 1994).

Os conhecimentos florístico e fitossociológico das florestas de terra firme da região são condições essenciais para a conservação de sua elevada diversidade. A obtenção e padronização dos atributos de diferentes ambientes florísticos e fisionômicos, são atividades básicas para a conservação e preservação, possibilitando a proposição de modelos mais adequados de manejo às florestas de terra firme na Amazônia Central, onde áreas protegidas são escassas e/ou menos eficientemente cuidadas.

\section{MATERIAL E MÉTODOS}

O estudo foi conduzido em um ambiente florestal de terra firme a noroeste da cidade de Manaus, Amazonas, com aproximadamente 6.000 ha, localizado na Estação Experimental ZF-2 do Instituto Nacional de Pesquisas da Amazônia, INPA, tendo como limites a Rodovia BR-174 (Manaus - Boa Vista) e a bacia do Rio Cuieiras (2035'45" S e 60¹2'40" W); a altitude do ambiente florestal varia de 40 a $100 \mathrm{~m}$.
Os solos sob a vegetação florestal se caracterizam por um gradiente textural, onde o horizonte superficial se apresenta mais arenoso do que o horizonte B. Em relação ao platô apresentam menores valores de bases trocáveis no horizonte superficial, bem como menor teor de matéria orgânica, nitrogênio e fósforo (Ranzani, 1980).

O clima é do tipo "Am" na classificação de Köppen (RADAM, 1978), com precipitação anual de 1.355 a 2.839 mm. A temperatura média oscila de 25,6 a $27,6{ }^{\circ} \mathrm{C}$, com umidade relativa do ar variando de 84 a $90 \%$ ao longo do ano; os meses mais chuvosos vão de Dezembro a Maio, e os mais secos de Agosto a Novembro.

Para o inventário florístico, amostrou-se dois transectos de 10 × $500 \mathrm{~m}$, paralelos e distantes $200 \mathrm{~m}$ entre si. Cada transecto foi dividido em 10 parcelas de $10 \times 50 \mathrm{~m}$, mensurando-se os indivíduos arbóreos, palmeiras e lianas com diâmetro à altura do peito (DAP) $\geqslant 10 \mathrm{~cm}$.

As amostras botânicas, férteis (flores e frutos) ou não, foram submetidas à secagem em estufa $\left(70^{\circ} \mathrm{C}\right)$ por 48 horas. Em seguida, essas amostras foram identificadas através da morfologia comparada com as exsicatas disponíveis no herbário do INPA (Cronquist, 1981) e de consultas à literatura especializada (Ribeiro et al., 1999). Após esse processo, o material fértil foi incorporado ao acervo do herbário do INPA; os estéreis serão mantidos por um período de até dois anos após a publicação dos resultados.

Os nomes botânicos apresentados foram conferidos com a página da WEB do Missouri Botanical Garden (http:// mobot.bobot.org/W3T/Search/vas.html). Aquelas amostras não identificadas com nomes científicos receberam códigos de morfotipo.

Os parâmetros fitossociológicos estimados foram: Densidade, Freqüência e Dominância Absolutas e Relativas, além dos Índices de Valor de Cobertura (IVC) e Importância (IVI), segundo Müller-Dombois \& Ellemberg (1974). Estimou-se ainda a importância ecológica das famílias no ecossistema a partir do Índice de Valor de Importância Familiar (IVIF), sendo este o resultado da soma da diversidade ( $\mathrm{n}^{\circ}$ de espécies da família $/ \mathrm{n}^{\circ}$ total de espécies), densidade e dominância relativas (Mori \& Boom, 1983).

O padrão de distribuição espacial dos indivíduos de cada espécie foi estimado segundo o índice de Morisita padronizado (Ip), desconsiderando da análise as espécies "localmente raras". As expressões matemáticas (Krebs, 1989) utilizadas para os cálculos foram:

a) $\mathrm{Id}=\mathrm{n}\left[\Sigma \mathrm{x}_{1}-\Sigma \mathrm{x}_{1} /\left(\Sigma \mathrm{x}_{1}\right)^{2}-\Sigma \mathrm{x}_{1}\right]$;

b) $\mathrm{Mu}=\chi_{0,975}^{2}-\mathrm{n}+\Sigma \mathrm{x}_{1} /\left(\sum \mathrm{x}_{1}\right)-1$;

c) $\mathrm{Ma}=\chi_{0,25}^{2}-\mathrm{n}+\Sigma \mathrm{x}_{1} /\left(\Sigma \mathrm{x}_{1}\right)-1$;

Se Id $\geqslant$ Ma $>1,0$, usa-se:

d) Ip $=0,5+0,5(\mathrm{Id}-\mathrm{Ma} / \mathrm{n}-\mathrm{Ma})$;

Se $\mathrm{Ma}>\mathrm{Id} \geqslant 1,0$, usa-se:

e) $\mathrm{Ip}=0,5(\mathrm{Id}-1 / \mathrm{Ma}-1)$; 


\section{ACTA AMAZONICA}

Se 1,0 > Id > Mu, usa-se:

f) Ip $=-0,5(\mathrm{Id}-1 / \mathrm{Mu}-1)$;

Se 1,0 > Mu > Id, usa-se:

g) Ip $=-0,5+0,5((\mathrm{Id}-\mathrm{Mu}) / \mathrm{Mu})$. Onde: Id = Índice de dispersão de Morisita; $\mathrm{n}=$ Número de parcelas; $\Sigma \mathbf{x}_{1}=$ Somatória do número de indivíduos da espécie i nas 20 parcelas $=\mathbf{x}_{1}+\mathbf{x}_{2}+\mathbf{x}_{3} \ldots+\mathbf{x}_{20} ; \Sigma \mathbf{x}_{1}^{2}=$ Somatória do quadrado do número de indivíduos da espécie i em cada parcela; $\mathrm{Mu}$ e $\mathrm{Ma}=$ Índices de uniformidade e agregação, respectivamente; $\chi_{0,975}^{2}$ e $\chi_{0,25}^{2}=$ Valores do Qui-quadrado tabelados com $n-1$ graus de liberdade que têm $97,5 \%$ e 2,5\% da área para a direita, respectivamente.

A interpretação do Índice padronizado de Morisita foi baseada no seguinte: caso o Ip $\geqslant 0,5$, o padrão espacial é agregado, se Ip $\leqslant-0,5$, o padrão é uniforme, e se for intermediário, é aleatório (Krebs, 1989).

As diversidades florísticas foram avaliadas através dos índices de Riqueza de Espécie e de Shannon-Wiener (Magurran, 1988). O primeiro índice $(\mathrm{S} / \mathrm{A})$ foi obtido da razão entre o número de espécies acumuladas e o tamanho da área amostrada $\left(\mathrm{m}^{2}\right)$, enquanto o segundo, pela fórmula: $H^{\prime}=-\Sigma\left(p_{i}\right)\left(\log _{e} p_{i}\right)$, onde $\mathrm{p}_{\mathrm{i}}=(\mathrm{ni} / \mathrm{N})$ é a probabilidade de que um indivíduo amostrado pertença a espécie $\mathrm{i} ; \mathrm{n}_{\mathrm{i}}=\mathrm{n}^{\mathrm{o}}$ total de indivíduos da espécie $\mathrm{i} ; \mathrm{N}=$ $\mathrm{n}^{\circ}$ total de indivíduos amostrados na área; a equitabilidade (uniformidade) foi obtida segundo a fórmula $E^{\prime}=H^{\prime} / \log _{e} S$ (Magurran, 1988), onde $\mathrm{S}=\mathrm{n}^{\circ}$ de espécies.

Calculou-se ainda o $\alpha$ de Fisher (Krebs, 1989) deste, e de outros trabalhos desenvolvidos em solos de terra firme, que forneceram o número de indivíduos amostrados e de espécies identificadas com diâmetro mínimo de inclusão $\geqslant 10 \mathrm{~cm}$. A fórmula utilizada foi: $S / N=1-x / x\left[-\log _{e}(1-x)\right]$, onde $S$ e $N$ $=$ Número total de espécies e indivíduos, respectivamente na amostra; $x=$ Parâmetro da série logarítmica; $a x, a x^{2} / 2, a x^{3} / 3$, $\mathrm{ax}^{4} / 4, \ldots$, onde $\mathrm{ax}=$ Número de espécies no total representada por um indivíduo; $\mathrm{ax}^{2} / 2=$ Número de espécies representada por dois indivíduos, e assim sucessivamente.

As similaridades florísticas entre as 20 parcelas foram estimadas através dos índices quantitativo de Porcentagem e qualitativo de Sorensen (Müeller-Dombois \& Ellemberg, 1974), com este último sendo calculado mediante a fórmula Cs $=(2 \mathrm{c} /(\mathrm{a}+\mathrm{b})) \times 100$, onde $\mathrm{c}=\mathrm{n}^{\circ}$ de espécies comuns às duas parcelas; $\mathrm{a}$ e $\mathrm{b}=\mathrm{n}^{\circ}$ de espécies presentes somente nas parcelas 1 e 2, respectivamente. As análises de agrupamento entre parcelas foram baseadas no método não ponderado pelas médias aritméticas (UPGMA) (Krebs, 1989), considerando inicialmente todas as espécies presentes na amostragem e em seguida, excluindo aquelas com apenas um indivíduo no hectare amostrado. Construiuse os dendogramas a partir dessas análises de agrupamento.

\section{RESULTADOS E DISCUSSÃO}

\section{Aspectos florísticos}

Foram registradas, entre os 771 indivíduos amostrados, 239 espécies distribuídas em 120 gêneros e 50 famílias botânicas. Dessas espécies, 201 foram determinadas em nível específico, 35 em nível de gênero, duas em nível de família e uma indeterminada (Tabela 1).

Tabela 1 - Relação das famílias, espécies, hábitos e seus interesses econômicos (comestível, madeireiro e medicinal) e/ou ecológico no ambiente florestal estudado. Manaus, Amazonas, Brasil.

\begin{tabular}{|c|c|c|c|}
\hline Famílias & Nomes Científicos & Hábitos & Interesses \\
\hline $\begin{array}{l}\text { Anacardiaceae } \\
\text { “ }\end{array}$ & $\begin{array}{l}\text { Anacardium giganteum Hancock ex Engl.* } \\
\text { Astronium sp.* }\end{array}$ & $\begin{array}{l}\text { árvore } \\
\text { “ }\end{array}$ & $\begin{array}{l}\text { ecológico } \\
\text { “ }\end{array}$ \\
\hline Annonaceae & Duguetia sp.* & “ & “ \\
\hline “ & D. chrysea Maas & “ & “ \\
\hline “ & D. surinamensis R.E.Fr. & “ & “ \\
\hline “ & Ephedranthus amazonicus R.E.Fr. & “ & madeireiro/ecológico \\
\hline “ & Guatteria sp. & “ & ecológico \\
\hline “ & G. discolor R.E.Fr. & “ & “ \\
\hline “ & G. olivacea R.E.Fr. & “ & “ \\
\hline “ & Rollinia insignis R.E.Fr.* & “ & “ \\
\hline “ & Xylopia sp.* & “ & “ \\
\hline “ & X. brasiliensis Spreng. ${ }^{*}$ & “ & “ \\
\hline Apocynaceae & Aspidosperma album (Vahl) R.Ben & “ & medicinal/ecológico \\
\hline “ & A. carapanauba Pichon.* & “ & “ \\
\hline “ & Geissospermum argenteum Woodson & “ & ecológico \\
\hline $\begin{array}{l}\text { Arecaceae } \\
\text { “ }\end{array}$ & $\begin{array}{l}\text { Oenocarpus bacaba Mart. } \\
\text { Syagrus inajai (Spruce) Becc.* }\end{array}$ & $\begin{array}{l}\text { palmeira } \\
\text { “ }\end{array}$ & $\begin{array}{l}\text { comestível/ecológico } \\
\text { ecológico }\end{array}$ \\
\hline
\end{tabular}




\section{ACTA \\ AMAZONICA}

FLORÍSTICA E FITOSSOCIOLOGIA DE UMA FLORESTA

DE VERTENTE NA AMAZÔNIA CENTRAL, AMAZONAS, BRASIL

\begin{tabular}{|c|c|c|c|}
\hline Famílias & Nomes Científicos & Hábitos & Interesses \\
\hline Bignoniaceae & Jacaranda copaia (Aubl.) D.Don & árvore & madeireiro/ecológico \\
\hline Bombacaceae & Scleronema micranthum Ducke & “ & 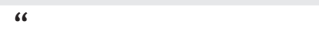 \\
\hline Boraginaceae & Cordia fallax Johnston. & “ & ecológico \\
\hline Burseraceae & Protium apiculatum Swart & “ & medicinal/ecológico \\
\hline “ & P. cf. carnosum Sm. & “ & “ \\
\hline “ & P. cf. decandrum (Aubl.) March. & “ & “ \\
\hline “ & P. cf. polybotryum (Turcz.) Engl.* & “ & “ \\
\hline “ & P. guianense (Aubl.) Marchand* & “ & “ \\
\hline “ & P. hebetatum Daly & “ & “ \\
\hline “ & P. insigne Engl. & “ & “ \\
\hline “ & P. strumosum Daly & “ & “ \\
\hline “ & P. trifoliolatum Engl. & “ & “ \\
\hline “ & Tetragastris cf. altissima (Aubl.) Swart* & “ & “ \\
\hline Caesalpiniaceae & Bauhinia sp.* & liana & ecológico \\
\hline “ & B. alata Ducke & “ & “ \\
\hline “ & B. cinnamomea DC.* & “ & “ \\
\hline “ & Dialium guianense Steud. ${ }^{*}$ & árvore & “ \\
\hline “ & Eperua duckeana R.S.Cowan & “ & “ \\
\hline “ & E. grabriflora (Ducke) R.S.Cowan & “ & “ \\
\hline “ & Macrolobium cf. acaciifolium Benth.* & “ & “ \\
\hline “ & M. cf. multijugum (DC.) Benth.* & “ & “ \\
\hline “ & M. gracile Spruce ex Benth. & “ & “ \\
\hline “ & M. limbatum Spruce ex Benth. & “ & “ \\
\hline “ & Peltogyne paniculata Benth. & “ & madeireiro/ecológico \\
\hline “ & Sclerolobium sp. $1^{*}$ & “ & “ \\
\hline “ & Sclerolobium sp. 2 & “ & “ \\
\hline “ & Sclerolobium eriopetalum Ducke & “ & “ \\
\hline “ & Tachigali sp.* & “ & “ \\
\hline “ & Tachigali myrmecophila Ducke* & “ & “ \\
\hline “ & Vouacapoua pallidior Ducke* & “ & “ \\
\hline Caryocaraceae & Caryocar glabrum ( Aubl.) Pers.* & “ & comestível/ecológico \\
\hline Cecropiaceae & Cecropia distachya Huber* & “ & ecológico \\
\hline “ & C. sciadophylla Mart. & “ & “ \\
\hline “ & Pourouma guianensis Aubl. & “ & “ \\
\hline “ & P. ovata Trécul & “ & “ \\
\hline “ & P. villosa Trécul* & “ & “ \\
\hline Celastraceae & Goupia glabra Aubl. & “ & comestível/ecológico \\
\hline Chrysobalanaceae & Couepia bracteosa Benth. & “ & ecológico \\
\hline “ & C. canomensis (Mart.) Benth. ex Hook & “ & “ \\
\hline “ & C. elata Ducke* & “ & “ \\
\hline “ & C. longipendula Pilg. & “ & “ \\
\hline “ & Hirtella araguariensis Prance* & “ & “ \\
\hline “ & H. bicornis Mart. \& Zucc.* & “ & “ \\
\hline “ & Licania canescens Benoist & “ & “ \\
\hline “ & L. cf. apetala (E. Mey.) Fritsch* & “ & “ \\
\hline “ & L. davillifolia Benoist & “ & “ \\
\hline “ & L. densiflora Kleinhoonte & “ & “ \\
\hline
\end{tabular}




\section{ACTA AMAZONICA}

\begin{tabular}{|c|c|c|c|}
\hline Famílias & Nomes Científicos & Hábitos & Interesses \\
\hline “ & L. heteromorpha Benth. & “ & “ \\
\hline “ & L. longistyla (Hook) Fritsch & “ & “ \\
\hline “ & L. micrantha Miq. & “ & “ \\
\hline “ & L. oblongifolia Standl. & “ & “ \\
\hline “ & L. octandra (Hoff. ex R. \& S) Prance & “ & madeireiro/ecológico \\
\hline Clusiaceae & Caraipa sp.* & “ & ecológico \\
\hline “ & Symphonia globulifera L. & “ & comestível/ecológico \\
\hline “ & Vismia japurensis Reichardt* & “ & ecológico \\
\hline Combretaceae & Buchenavia parvifolia Ducke & “ & madeireiro/ecológico \\
\hline Connaraceae & Connarus perrottetti (DC.) Planch.* & “ & ecológico \\
\hline “ & Rourea cuspidata Benth. ex Backer* & liana & “ \\
\hline Dichapetalaceae & Tapura amazonica Poepp. \& Endl.* & árvore & “ \\
\hline Ebenaceae & Diospyros pseudoxylopia Mildbr. & “ & “ \\
\hline Elaeocarpaceae & Sloanea cf. guianensis (Aubl.) Benth. & “ & “ \\
\hline “ & S. excelsa Ducke & “ & “ \\
\hline “ & S. floribunda Spruce ex Benth. & “ & “ \\
\hline Euphorbiaceae & Alchornea sp.* & “ & “ \\
\hline “ & Conceveiba guianensis Aubl. & “ & “ \\
\hline “ & Croton lanjouwensis Jablonski* & “ & “ \\
\hline “ & Hevea guianensis Aubl. & “ & “ \\
\hline “ & Mabea sp. 1 & “ & “ \\
\hline “ & Mabea sp. 2 & “ & “ \\
\hline “ & M. angularis D. den Hollander & “ & “ \\
\hline “ & M. caudata Pax. \& K.Hoffm. & “ & “ \\
\hline “ & M. cf. angustifolia Spruce ex Benth. & “ & “ \\
\hline “ & M. cf. klugii Steyerm & “ & “ \\
\hline “ & M. piriri Aubl. & “ & “ \\
\hline “ & M. speciosa Mull.Arg. & “ & “ \\
\hline “ & Micrandropsis scleroxylon W. Rod. & “ & madeireiro/ecológico \\
\hline “ & Senefeldera macrophylla Ducke* & “ & ecológico \\
\hline Fabaceae & Andira micrantha Ducke* & “ & “ \\
\hline “ & Bocoa viridiflora (Ducke) R.S.Cowan & “ & “ \\
\hline “ & Fabaceae indeterminada & “ & ecológico \\
\hline “ & Ormosia grossa Rudd* & “ & “ \\
\hline “ & O. costulata (Miq.) Kleinhoonte* & “ & “ \\
\hline “ & Pterocarpus sp.* & “ & madeireiro/ecológico \\
\hline “ & P. rohrii Vahl* & “ & “ \\
\hline “ & Stryphnodendron microstachyum Poepp. & “ & ecológico \\
\hline “ & Swartzia arborescens (Aubl.) Pittier* & “ & “ \\
\hline “ & S. cf. recurva Poepp. \& Endl. & “ & “ \\
\hline “ & S. dolichopoda R.S. Cowan & “ & “ \\
\hline “ & S. ingifolia Ducke & “ & “ \\
\hline “ & S. longistipitata Ducke* & “ & “ \\
\hline “ & S. polyphylla DC. & “ & “ \\
\hline “ & S. reticulata Ducke & “ & “ \\
\hline “ & S. schomburgkii Benth. & “ & “ \\
\hline “ & S. ulei Harms & “ & “ \\
\hline
\end{tabular}




\section{ACTA \\ AMAZONICA}

\begin{tabular}{|c|c|c|c|}
\hline Famílias & Nomes Científicos & Hábitos & Interesses \\
\hline “ & Vatairea sericea Ducke* & “ & “ \\
\hline \multirow{2}{*}{$\begin{array}{l}\text { Flacourtiaceae } \\
\text { “ }\end{array}$} & Casearia cf. javitensis H.B.K.* & “ & “ \\
\hline & C. combaymensis Tul.* & “ & “ \\
\hline Humiriaceae & Duckesia verrucosa (Ducke) Cuatr.* & “ & madeireiro/ecológico \\
\hline “ & Sacoglottis guianensis Benth. & “ & “ \\
\hline “ & Vantanea parviflora Lam. & “ & ecológico \\
\hline Icacinaceae & Humirianthera rupestris Ducke & “ & “ \\
\hline Indeterminada & Indeterminada sp.* & “ & “ \\
\hline Lauraceae & Aniba canelilla (H.B.K.) Mez ${ }^{*}$ & “ & medicinal/ecológico \\
\hline “ & A. roseaodora Ducke* & “ & “ \\
\hline “ & Licaria sp.* & “ & madeireiro/ecológico \\
\hline “ & L. cannela (Meissn.) Kosterm. & “ & “ \\
\hline “ & L. guianensis Aubl.* & “ & “ \\
\hline “ & Mezilaurus sp.* & “ & “ \\
\hline “ & M. duckei van der Werff* & “ & “ \\
\hline “ & M. itauba (Meissn.) Taub. ex Mez & “ & “ \\
\hline “ & Ocotea cajumari Mart. & “ & “ \\
\hline “ & O. cf. longifolia H.B.K* & “ & “ \\
\hline “ & O. cinerea van der Werff & “ & “ \\
\hline “ & O. guianensis Aubl. & “ & “ \\
\hline “ & O. tabacifolia (Meiss.) Roher* & “ & “ \\
\hline Lecythidaceae & Cariniana sp.* & “ & madeireiro/ecológico \\
\hline “ & C. decandra Ducke & “ & “ \\
\hline “ & Conceveiba guianensis Aubl. & “ & “ \\
\hline “ & Corythophora sp. & “ & madeireiro/ecológico \\
\hline “ & C. alta Knuth & “ & “ \\
\hline “ & Couratari stellata A.C.Sm.* & “ & ecológico \\
\hline “ & Eschweilera sp.* & “ & “ \\
\hline “ & E. amazonica Knuth & “ & “ \\
\hline “ & E. amazoniciformis Mori* & “ & “ \\
\hline “ & E. atropetiolata Mori & “ & madeireiro/ecológico \\
\hline “ & E. bracteosa (Poepp. \& Endl.) Miers & “ & ecológico \\
\hline “ & E. collina Eyma & “ & “ \\
\hline “ & E. coriacea (DC.) Mart. ex Berg. & “ & “ \\
\hline “ & E. odora (Poepp.) Miers. & “ & “ \\
\hline “ & E. tessmannii Knuth & “ & “ \\
\hline “ & Gustavia augusta L.* & “ & “ \\
\hline “ & G. hexapetala (Aubl.) Sm. & “ & “ \\
\hline “ & Lecythis sp.* & “ & madeireiro/ecológico \\
\hline “ & L. barnebyi Mori* & “ & “ \\
\hline “ & L. graciena Mori & “ & “ \\
\hline “ & L. prancei Mori* & “ & “ \\
\hline Malpighiaceae & Byrsonima chrysophylla H.B.K.* & “ & ecológico \\
\hline Melastomataceae & Miconia argyrophylla DC.* & “ & “ \\
\hline Meliaceae & Guarea carinata Ducke* & “ & “ \\
\hline “ & G. convergens T.D.Penn.* & “ & “ \\
\hline “ & G. humaitensis T.D.Penn. & “ & “ \\
\hline
\end{tabular}




\section{ACTA AMAZONICA}

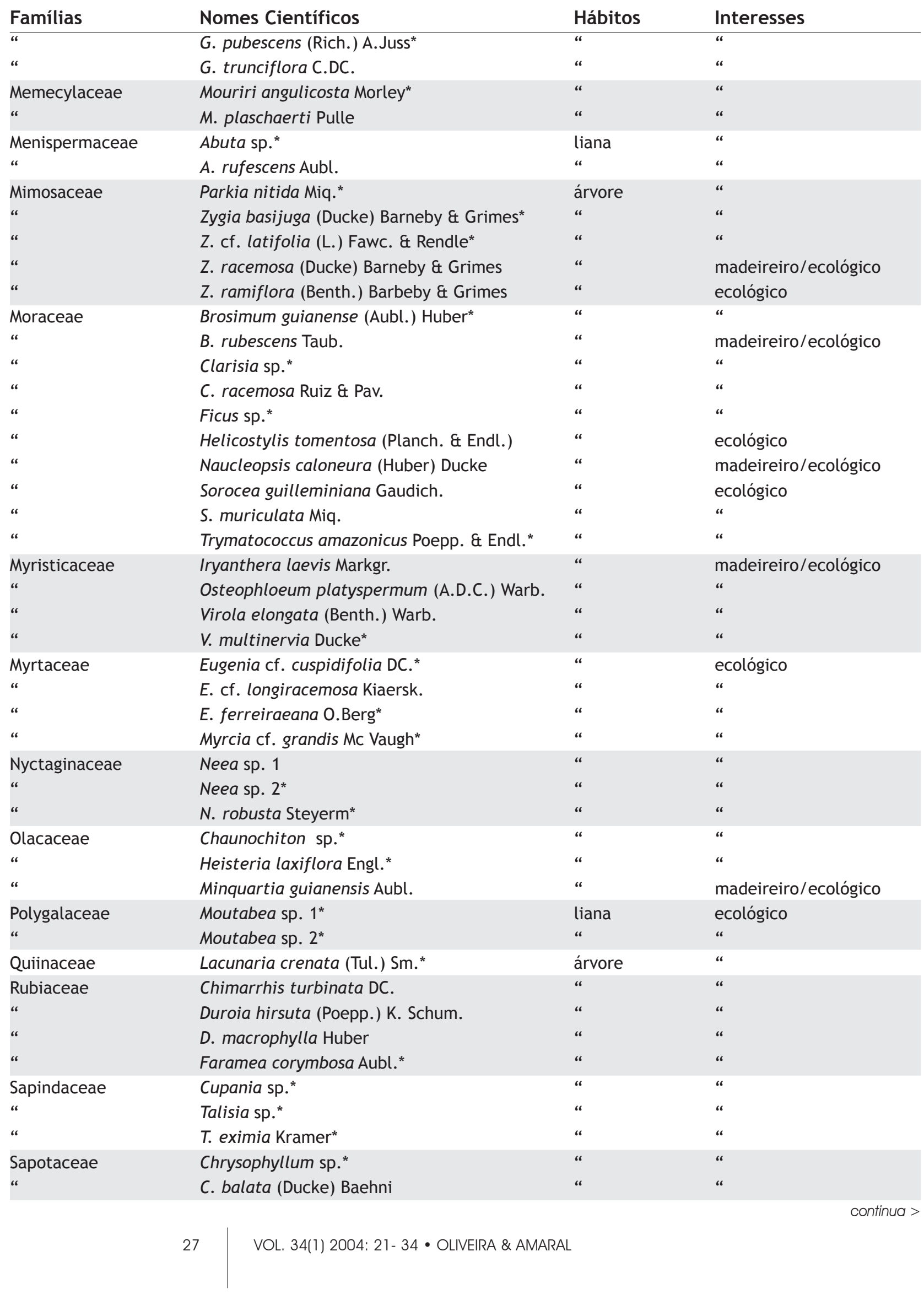




\section{ACTA AMAZONICA}

\begin{tabular}{|c|c|c|c|}
\hline Famílias & Nomes Científicos & Hábitos & Interesses \\
\hline$\overline{~ “ ~}$ & C. prieurii A.D.C. & “ & “ \\
\hline “ & Ecclinusa guianensis Eyma & “ & “ \\
\hline “ & Manilkara huberi (Ducke) A.Chev. ${ }^{*}$ & “ & madeireiro/ecológico \\
\hline “ & Micropholis sp.* & “ & 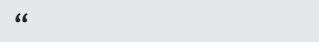 \\
\hline “ & M. casiquiarensis Aubrév. & “ & “ \\
\hline “ & M. guyanensis (A.D.C) Pierre* & “ & “ \\
\hline “ & M. trunciflora Ducke* & “ & “ \\
\hline “ & M. williamii Aubrév. \& Pellegrin & “ & “ \\
\hline “ & Pouteria sp. $1^{*}$ & “ & “ \\
\hline “ & Pouteria sp. 2 & “ & “ \\
\hline “ & P. anomala (Pires) T.D.Penn. & “ & “ \\
\hline “ & P. bilocularis (Winkler) Baehni & “ & “ \\
\hline “ & P. campanulata Baehni & “ & “ \\
\hline “ & P. cf. cladantha Sandwith & “ & “ \\
\hline “ & P. erythrochrysa T.D.Penn.* & “ & “ \\
\hline “ & P. filipes Eyma & “ & “ \\
\hline “ & P. freitasii T.D.Penn. & “ & “ \\
\hline “ & P. glomerata (Miq.) Baehni & “ & “ \\
\hline “ & P. gongrijpii Eyma* & “ & “ \\
\hline “ & P. guianensis Aubl. & “ & comestível/ecológico \\
\hline “ & P. platyphylla (A.C.Sm.) Baehni & “ & ecológico \\
\hline Simaroubaceae & Simarouba amara Aubl. & “ & madeireiro/ecológico \\
\hline \multirow{2}{*}{$\begin{array}{l}\text { Siparunaceae } \\
\text { “ }\end{array}$} & Siparuna decipiens (Tul.) A.D.C* & “ & ecológico \\
\hline & S. guianensis Aubl. & “ & “ \\
\hline \multirow{2}{*}{$\begin{array}{l}\text { Sterculiaceae } \\
\text { “ }\end{array}$} & Sterculia excelsa Mart.* & “ & “ \\
\hline & Theobroma sylvestre Mart. & “ & “ \\
\hline Tiliaceae & Lueheopsis rosea (Ducke) Burret & “ & “ \\
\hline Ulmaceae & Ulmaceae indeterminada * & “ & “ \\
\hline Violaceae & Amphyrrhox sp.* & “ & “ \\
\hline “ & Leonia glycycarpa Ruiz \& Pav. & “ & “ \\
\hline “ & Paypayrola sp. & “ & “ \\
\hline “ & Rinorea guianensis Aubl. & “ & “ \\
\hline “ & R. racemosa (Mart.) Kuntze & “ & “ \\
\hline \multirow{2}{*}{$\begin{array}{l}\text { Vochysiaceae } \\
\text { “ }\end{array}$} & Erisma bicolor Ducke* & “ & “ \\
\hline & Qualea paraensis Ducke & “ & “ \\
\hline
\end{tabular}

* Espécies "localmente raras" no hectare estudado.

Considerando como espécies "localmente raras" aquelas que ocorrem na amostragem com apenas um indivíduo (Oliveira $e t$ al., 2003), pode-se inferir que neste ambiente florestal, $44 \%$ das espécies são raras (105), corroborando outros estudos na região (Porto et al., 1976; Prance et al., 1976; Amaral, 1996; Oliveira, 1997; Oliveira et al., 2003), onde foram encontrados entre 40 a $60 \%$ dessas espécies em levantamentos florísticos de 1 hectare.

Os gêneros com maior riqueza florística foram Pouteria, com 13 espécies; Eschweilera, Licania, Protium e Swartzia, com 9; e Mabea, com 8. Conjuntamente, esses seis gêneros representaram $24 \%$ das espécies registradas.
As famílias com maior número de espécies foram Sapotaceae, com 23 espécies; Lecythidaceae, com 21; Fabaceae, com 18; Caesalpiniaceae, com 17; Chrysobalanaceae, com 15; e Euphorbiaceae, com 14. Essas seis famílias contribuíram com $45 \%$ da riqueza local, sugerindo que a diversidade vegetal concentra-se em poucas famílias botânicas, ratificando outros estudos na região (Jardim \& Hosokawa, 1986/1987; Matos \& Amaral, 1999; Lima Filho et al., 2001; Oliveira et al., 2003). Lauraceae, com 13; Annonaceae, Burseraceae e Moraceae, cada uma com 10 espécies, completam a lista das dez famílias mais 


\section{ACTA \\ AMAZONICA}

diversificadas presentes nesse ecossistema de terra firme. Por outro lado, 32\% das famílias expressaram apenas uma espécie; não que elas sejam monoespecíficas (Ribeiro et al., 1999), mas porque neste ambiente florestal, possivelmente, são famílias constituídas de poucas espécies, portanto, mais difíceis de serem encontradas.

Excetuando-se a riqueza de espécies para o gênero Mabea (Oliveira et al., 2003), o perfil florístico observado neste estudo é típico das florestas de terra firme da Amazônia Central (Jardim \& Hosokawa, 1986/1987; Tello, 1995; Matos \& Amaral, 1999; Amaral et al., 2000; Lima Filho et al., 2001).

Comparando o número de indivíduos com outros estudos no Estado do Amazonas (Tello, 1995; Matos \& Amaral, 1999; Oliveira \& Mori, 1999; Amaral et al., 2000; Lima Filho et al. 2001; Oliveira et al., 2003), notou-se que a área amostrada possui maior densidade de espécimes por hectare. Contudo, essa alta densidade não se traduziu em alta diversidade florística, conforme observado em outros trabalhos na região (Amaral, 1996; Oliveira \& Mori, 1999; Lima Filho et al., 2001).

As famílias mais importantes, em ordem decrescente no número de indivíduos, foram: Lecythidaceae (118), Burseraceae (87), Sapotaceae (78), Chrysobalanaceae (72), Euphorbiaceae (62) e Fabaceae (43). Juntas, essas seis famílias responderam por quase $60 \%$ dos espécimes inventariados, ficando as 44 famílias restantes, responsáveis por $40,3 \%$ do total, evidenciando a baixa abundância relativa de indivíduos nestas famílias (Figura 1).

A estrutura diamétrica evidenciou que mais de $65 \%$ dos indivíduos amostrados encontram-se distribuídos na primeira classe de diâmetro (Figura 2). Essa constatação deve-se à dinâmica natural de mortalidade e recrutamento de novos indivíduos à comunidade em decorrência de quedas de árvores nesse ambiente florestal, o que refletiria inclusive sobre a diversidade local de espécies (Phillips et al., 1994). Uma outra explicação envolve as espécies próprias de DAP menores, como as palmeiras e lianas lenhosas, relativamente freqüentes nessa comunidade vegetal (Tabela 1).

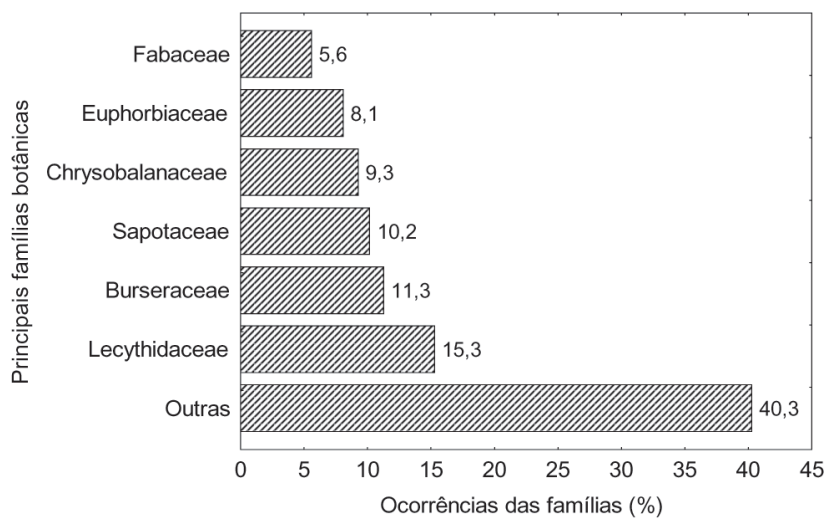

Figura 1 - Distribuição percentual da diversidade de plantas nas principais famílias botânicas amostradas no ambiente florestal estudado. Manaus, Amazonas, Brasil.

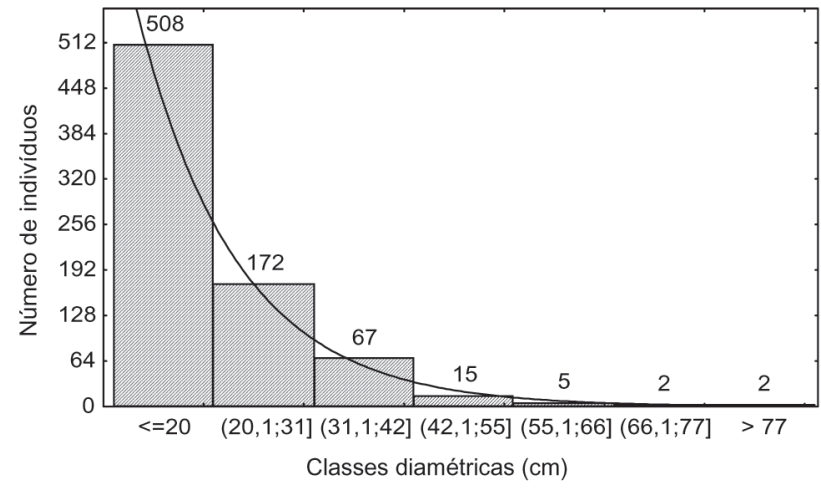

Figura 2 - Classes de diâmetro (DAP $\geqslant 10 \mathrm{~cm}$ ) dos 771 indivíduos amostrados no ambiente florestal estudado. Manaus, Amazonas, Brasil.

Para vários autores (Jardim \& Hosokawa, 1986/1987; Jardim, 1995; Tello, 1995; Oliveira \& Mori, 1999; Amaral et al., 2000; Lima Filho et al., 2001; Oliveira et al., 2003) a distribuição diamétrica obtida neste estudo (maior número de indivíduos na primeira classe de DAP) é típico de muitas florestas nativas da Amazônia Central. Porém, essa tendência de "J invertido" também tem sido observada em florestas secundárias ou em florestas no início da sucessão (Rabelo et al., 2002; Santana, 2002).

Estudos recentes (Oliveira \& Mori, 1999; Oliveira et al., 2003) propõem que o comportamento decrescente da curva indica pouca ou nenhuma pressão antrópica sobre o ambiente florestal, o que é ratificado tanto pelo pequeno número de espécies pioneiras, como pela alta diversidade florística no hectare inventariado.

\section{ASPECTOS FITOSSOCIOLÓGICOS}

O Índice de Valor de Importância (IVI) estimado para as espécies vegetais, em áreas não perturbadas, pode ser utilizado em planos de manejo, como indicador da importância ecológica, devido à influência das espécies mais freqüentes e dominantes nos processos básicos de equilíbrio da flora $\mathrm{e}$ manutenção da fauna, fornecendo abrigo e alimentação.

Analisando-se as espécies amostradas quanto à sua importância ecológica, das dez figuradas com maior IVI, destacaram-se Eschweilera bracteosa, Protium apiculatum, Pouteria filipes, Eschweilera tessmannii e Licania octandra (Tabela 2). Eschweilera bracteosa, Pouteria filipes, Eschweilera tessmannii e Licania octandra destacaram-se pelo porte de seus indivíduos, proporcionando-lhes maior dominância. Por outro lado, Protium apiculatum possui a maior densidade de todas as espécies, justificando seu elevado índice registrado neste estudo; Eschweilera bracteosa (DR $=3,89 ; \mathrm{FR}=2,30 ; \mathrm{DoR}=3,78$ ) e Protium apiculatum (DR $=4,93 ; \mathrm{FR}=2,47 ; \mathrm{DoR}=2,45)$ são as espécies melhor distribuídas ao longo da área avaliada, uma vez que possuem parâmetros fitossociológicos relativamente uniformes (Matos \& Amaral, 1999). 


\section{ACTA \\ AMAZONICA}

FLORÍSTICA E FITOSSOCIOLOGIA DE UMA FLORESTA

DE VERTENTE NA AMAZÔNIA CENTRAL, AMAZONAS, BRASIL
As espécies Protium stromosum, Licania beteromorpha, Brosimum rubescens, Qualea paraensis, Eschweilera coriaceae, além das supracitadas, são comumente abordadas na maioria dos trabalhos desenvolvidos em ecossistemas de terra firme da Amazônia (Tello, 1995; Amaral, 1996; Oliveira, 1997; Matos \& Amaral, 1999; Amaral et al., 2000; Lima Filho, 2001; Oliveira et al., 2003), onde figuram entre as dez mais importantes quanto a esse índice.

As famílias com maiores Índices de Valor de Importância Familiar (IVIF) foram Lecythidaceae, Sapotaceae, Chrysobalanaceae, Burseraceae, Fabaceae, Euphorbiaceae, Caesalpiniaceae, Moraceae, Lauraceae e Annonaceae, todas produtoras de frutos (Figura 3). De um total máximo de 300\% de IVIF, essas dez famílias detiveram mais de $213 \%$, ratificando a importância ecológica das mesmas aos equilíbrios faunístico e florístico das florestas de terra firme da Amazônia Central (Matos \& Amaral, 1999; Oliveira et al., 2003).

Nota-se ainda que nem sempre a família com maior riqueza em espécies é a de maior importância na comunidade (Figura 3). A família Sapotaceae tida como a mais diversificada neste estudo, com 9,6\%, é a segunda em importância ecológica. Por outro lado, Burseraceae, com apenas 4,2\% da diversidade, manifestou a quarta maior importância ecológica em relação às outras famílias registradas. Estes resultados mostram que além da riqueza específica, as variáveis densidade e dominância relativas, especialmente estas duas últimas, são determinantes quando se avaliam as famílias florestais da região (Oliveira et al., 2003).

Excluindo as espécies "localmente raras" da análise de dispersão espacial, observou-se que $82,8 \%$ das espécies encontram-se distribuídas aleatoriamente na área amostrada. Com relação às 20 espécies com maior IVI, 15 delas seguiram padrão espacial aleatório (75\%), três padrão uniforme (15\%) e duas padrão agregado (10\%) (Tabela 2); as espécies gregárias foram Eschweilera tessmannii e E. coriacea (Tabela 2). Rossi \& Higuchi (1998) comparando distintos métodos de análise de distribuição espacial de algumas espécies arbóreas na Amazônia Central, também constataram predominância do padrão aleatório em seus estudos. A prevalência desse padrão de distribuição espacial pode sugerir influência mínima de

Tabela 2 - Relação das espécies presentes na floresta de vertente, Manaus, Amazonas, em ordem decrescente de IVI (Índice de Valor de Importância). $\mathrm{N}=$ número de indivíduos; $\mathrm{P}=$ número de parcelas onde ocorre; $\mathrm{DA}=$ densidade absoluta; $\mathrm{DR}=$ densidade relativa; FA = freqüência absoluta; $\mathrm{FR}=$ freqüência relativa; DoA = dominância absoluta; DoR = dominância relativa; $\mathrm{VC}=$ valor de cobertura; Ip = índice de Morisita padronizado.

\begin{tabular}{lccccccccccc}
\hline \hline Espécies & N & P & DA & DR & FA & FR & DoA & DoR & VC & IVI & Ip* $^{*}$ \\
\hline Eschweilera bracteosa & 30 & 14 & 30 & 3,89 & 70 & 2,30 & 1,15 & 3,78 & 7,67 & 9,97 & $-0,61$ \\
Protium apiculatum & 38 & 15 & 38 & 4,93 & 75 & 2,47 & 0,74 & 2,45 & 7,37 & 9,84 & $-0,51$ \\
Pouteria filipes & 24 & 12 & 24 & 3,12 & 60 & 1,97 & 1,24 & 4,10 & 7,21 & 9,18 & $-0,35$ \\
Eschweilera tessmannii & 24 & 11 & 24 & 3,11 & 55 & 1,81 & 1,18 & 3,89 & 7,01 & 8,82 & 0,51 \\
Licania octandra & 21 & 12 & 21 & 2,72 & 60 & 1,97 & 1,00 & 3,29 & 6,01 & 7,99 & $-0,05$ \\
Protium strumosum & 23 & 11 & 23 & 2,98 & 55 & 1,81 & 0,43 & 1,43 & 4,41 & 6,22 & $-0,51$ \\
Licania heteromorpha & 12 & 10 & 12 & 1,56 & 50 & 1,64 & 0,72 & 2,39 & 3,95 & 5,59 & 0,21 \\
Brosimum rubescens & 8 & 8 & 8 & 1,04 & 40 & 1,32 & 0,73 & 2,42 & 3,45 & 4,77 & 0,35 \\
Qualea paraensis & 4 & 4 & 4 & 0,52 & 20 & 0,66 & 0,94 & 3,05 & 3,57 & 4,23 & 0,15 \\
Eschweilera coriacea & 12 & 5 & 12 & 1,56 & 25 & 0,82 & 0,44 & 1,46 & 3,02 & 3,84 & 0,53 \\
Hevea guianensis & 8 & 7 & 8 & 1,04 & 35 & 1,15 & 0,46 & 1,52 & 2,56 & 3,71 & 0,10 \\
Eschweilera amazonica & 7 & 5 & 7 & 0,91 & 25 & 0,82 & 0,47 & 1,55 & 2,46 & 3,28 & $-0,27$ \\
Chrysophyllum prieurii & 6 & 6 & 6 & 0,78 & 30 & 0,99 & 0,44 & 1,47 & 2,24 & 3,23 & 0,25 \\
Eschweilera atropetiolata & 9 & 7 & 9 & 1,17 & 35 & 1,15 & 0,25 & 0,83 & 2,00 & 3,15 & $-0,04$ \\
Eschweilera collina & 6 & 5 & 6 & 0,78 & 25 & 0,82 & 0,43 & 1,41 & 2,18 & 3,01 & $-0,08$ \\
Swartzia reticulata & 8 & 8 & 8 & 1,04 & 40 & 1,32 & 0,17 & 0,55 & 1,59 & 2,90 & 0,36 \\
Oenocarpus bacaba & 9 & 8 & 9 & 1,17 & 40 & 1,32 & 0,12 & 0,39 & 1,56 & 2,87 & 0,18 \\
Guarea humaitensis & 7 & 6 & 7 & 0,91 & 30 & 0,99 & 0,29 & 0,97 & 1,86 & 2,86 & 0,01 \\
Corythophora alta & 6 & 6 & 6 & 0,78 & 30 & 0,99 & 0,32 & 1,06 & 1,84 & 2,82 & 0,25 \\
Scleronema micranthum & 7 & 5 & 7 & 0,91 & 25 & 0,82 & 0,33 & 1,09 & 2,00 & 2,82 & $-0,27$ \\
\hline Sub-Totais & 269 & 165 & 269 & 34,89 & 825 & 27,14 & 11,86 & 39,07 & 101,10 & 73,96 & - \\
Outras espécies & 502 & 443 & 502 & 65,11 & 2215 & 72,86 & 18,49 & 60,93 & 98,90 & 226,04 & - \\
\hline Totais & 771 & 608 & 771 & 100 & 3040 & 100 & 30,34 & 100 & 200 & 300 & - \\
\hline \hline
\end{tabular}

*Ip ${ }^{3} 0,5$ - distribuição agregada; Ip £ -0,5 - uniforme; Ip intermediário (-0,5 a 0,5) - aleatória. 


\section{ACTA \\ AMAZONICA}

fatores abióticos (características edáficas, disponibilidade de espaço, luz e/ou água) e uma maior influência de fatores bióticos (competição intra e interespecífica, comportamento reprodutivo das espécies, dispersão dos frutos ou herbivoria), na distribuição horizontal das populações.

\section{ASPECTOS ECOLÓGICOS}

A curva espécie-área indicou que a comunidade vegetal é bastante heterogênea quanto à composição florística e, apesar disso, insuficientemente amostrada (Figura 4), uma vez que esta não se estabilizou em apenas 1 hectare, ratificando outros estudos documentados para a Amazônia Central (Porto et al., 1976; Tello, 1995; Amaral, 1996; Amaral et al., 2000).

$\mathrm{O}$ índice de Shannon-Wiener para florestas tropicais normalmente varia de 3,83 a 5,85, valores considerados altos para qualquer tipo de vegetação (Knight, 1975). Diante disso, pode-se concluir que o ambiente florestal estudado possui grande diversidade florística, pois apresentou índice de 5,01, corroborando a alta diversidade estimada através da curva espécie-área (Figura 4).

Quando se compara aos índices de outras florestas da região, com $\log _{\mathrm{e}}$ e DAP mínimo de $10 \mathrm{~cm}$ (Porto et al., 1976; Tello, 1995, Higuchi et al., 1998), pode-se inferir que o índice observado neste estudo é um dos maiores já documentados para a Amazônia Central, sendo superado somente pelas comunidades vegetais de platô da ZF-2 (Oliveira et al., 2003) e do Rio Urucu (Amaral, 1996), onde foram registrados valores de 5,10 e 5,28, respectivamente.

Em virtude do índice de Shannon-Wiener ter sido reportado em poucos trabalhos com DAP de inclusão mínimo de $10 \mathrm{~cm}$, decidiu-se estimar o $\alpha$ de Fisher para fins de comparação com outros ambientes florestais de terra firme da Amazônia Central (Tabela 3).

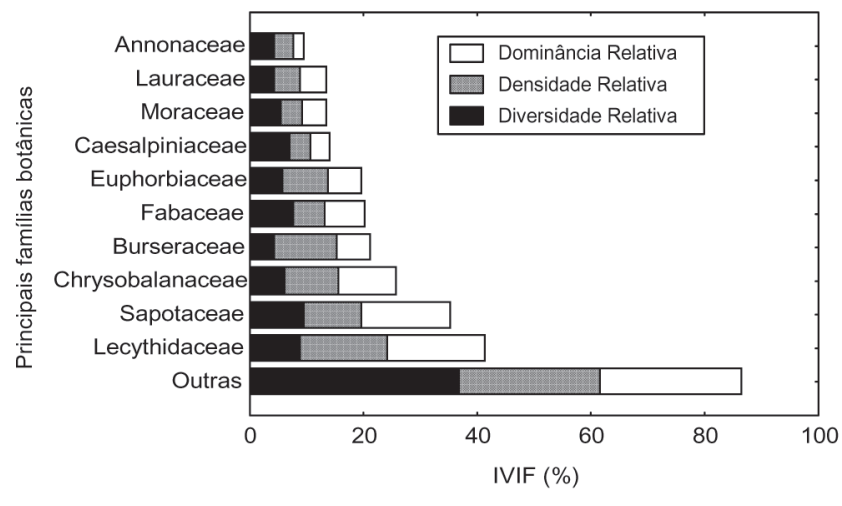

Figura 3 - Composição do Índice de Valor de Importância Familiar (IVIF) das dez famílias botânicas mais importantes amostradas no ambiente florestal estudado. Manaus, Amazonas, Brasil.

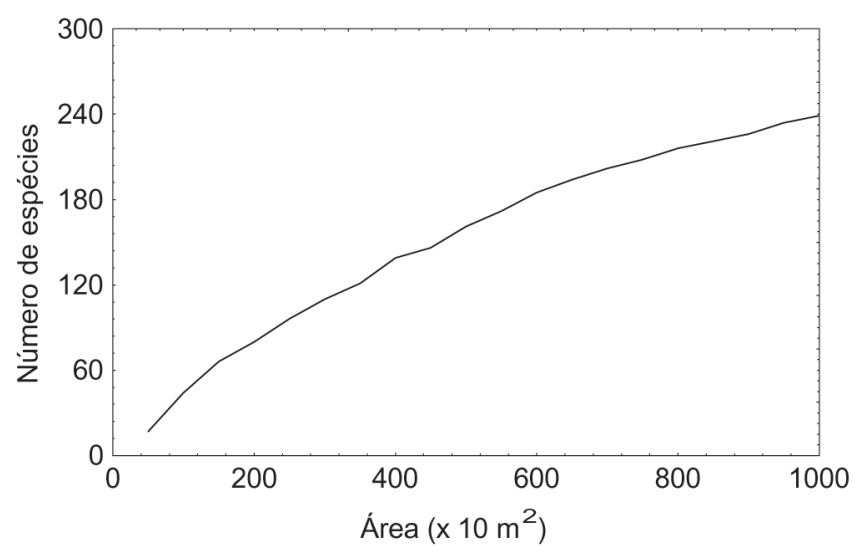

Figura 4 - Curva cumulativa das espécies com $\mathrm{DAP}^{3} 10 \mathrm{~cm}$ amostradas no ambiente florestal estudado. Manaus, Amazonas, Brasil.

Tabela 3 - Comparação da diversidade florística entre a área estudada e outras localidades de terra firme na Amazônia Central. Manaus, Amazonas, Brasil.

\begin{tabular}{|c|c|c|c|c|c|c|}
\hline Localidades/Coordenadas & $\mathrm{Nf}$ & $\mathrm{Ng}$ & Ns & $\mathrm{Ni}$ & $\alpha$ & Autores \\
\hline $\begin{array}{l}\text { Reserva Ducke } \\
\left(03^{\circ} 08^{\prime} \mathrm{S}, 60^{\circ} 02^{\prime} \mathrm{W}\right)\end{array}$ & 44 & 103 & 142 & 719 & 53,01 & Tello (1995) \\
\hline $\begin{array}{l}\text { Região do Rio Urucu } \\
\left(4^{\circ} 51^{\prime}-4^{\circ} 52^{\prime} \text { S, 65⒈' }-65^{\circ} 20^{\prime} \text { W) }\right.\end{array}$ & 46 & 126 & 253 & 710 & 140,51 & Amaral (1996) \\
\hline $\begin{array}{l}\text { Entre Estrada da Várzea e o Rio Uatumã } \\
\left(2^{\circ} 22^{\prime}-2^{\circ} 45^{\prime} \mathrm{S}, 58^{\circ} 15^{\prime}-58^{\circ} 31^{\prime} \mathrm{W}\right)\end{array}$ & 47 & 118 & 196 & 527 & 108,71 & Matos \& Amaral (1999) \\
\hline $\begin{array}{l}\text { Região próxima à Manaus } \\
\left(2^{\circ} 24^{\prime}-2^{\circ} 25^{\prime} \text { S, 5943’ - } 59^{\circ} 45 \mathrm{~W}\right)\end{array}$ & 47 & 138 & 285 & 618 & 205,08 & Oliveira \& Mori (1999) \\
\hline $\begin{array}{l}\text { Região do Rio Uatumã } \\
\left(2^{\circ} 20^{\prime} \text { S, } 58^{\circ} 45^{\prime}\right)\end{array}$ & 47 & 118 & 145 & 741 & 54,71 & Amaral et al. (2000) \\
\hline $\begin{array}{l}\text { Região próxima à Coari } \\
\left(4^{\circ}-5^{\circ} \mathrm{S}, 63^{\circ}-66^{\circ} \mathrm{W}\right)\end{array}$ & 48 & 122 & 269 & 762 & 208,31 & Lima Filho et al. (2001) \\
\hline
\end{tabular}

$\mathrm{Nf}=\mathrm{N}$ úmero de famílias; $\mathrm{Ng}=\mathrm{N}$ úmero de gêneros; $\mathrm{N} \mathrm{s}=\mathrm{N}$ úmero de espécies; $\mathrm{Ni}=\mathrm{N}$ úmero de indivíduos; $\alpha=\mathrm{Alfa}$ de Fisher. 


\section{ACTA AMAZONICA}

FLORÍSTICA E FITOSSOCIOLOGIA DE UMA FLORESTA

DE VERTENTE NA AMAZÔNIA CENTRAL, AMAZONAS, BRASIL
Os valores de $\alpha$ de Fisher variaram de 53,01 ( $\mathrm{N}=741$ e $\mathrm{S}$ $=145=)$ a $208,3(\mathrm{~N}=769$ e $\mathrm{S}=322)$. Observou-se que a alfa-diversidade obtida neste estudo $(118,62)$ é inferior a de outras regiões próximas aos Municípios de Manaus (Oliveira \& Mori, 1999) e Coari (Lima Filho et al., 2001), porém, superior aos valores registrados para as demais comunidades vegetais (Tabela 3 ). Notou-se ainda que, excetuando o $\alpha$ de Fisher de 53,01 para a vegetação da Reserva Ducke (Tello, 1995), as demais comunidades sobre relevos mais ondulados (declives) apresentaram grande número de espécies em relação ao de indivíduos amostrados (Amaral, 1996; Oliveira \& Mori, 1999; Lima Filho et al., 2000), resultando em algumas das maiores alfas-diversidades já documentadas para as florestas de terra firme da Amazônia (Steege, 2000). Estes resultados podem estar associados à gradientes edáficos e topográficos, como também a maior taxa de mortalidade e recrutamento de novos indivíduos e/ou espécies à comunidade florestal, como anteriormente discutido.

O grau estimado de equitabilidade de 0,91 sugere alta uniformidade nas proporções indivíduos/espécies dentro da comunidade vegetal, constatação esperada, pois a equitabilidade é diretamente proporcional à diversidade e, antagônico à dominância (Uhl \& Murphy, 1981). A alta heterogeneidade florística refletida a partir da alta equitabilidade indica claramente que não ocorre dominância de uma ou poucas espécies nas florestas nativas da Amazônia.

Estudos florísticos conduzidos em dois transectos de $20 \mathrm{x}$ $2500 \mathrm{~m}$, na mesma área experimental, revelou equitabilidade de 0,60 , caracterizando distribuição moderadamente uniforme dos indivíduos entre as espécies identificadas (Higuchi et al., 1998). Essa diferença na distribuição pode ser explicada, pelo menos em parte, pelo tamanho da amostragem, onde a proporção indivíduo/espécie tenderia a ser mais uniforme em parcelas menores (Oliveira et al., 2003).

O maior e o menor índice de similaridade obtidos entre as 20 parcelas de $500 \mathrm{~m}^{2}$, foram, respectivamente, 36, 1 e $22,6 \%$ para o coeficiente quantitativo de Porcentagem (Figura 5a). Nas parcelas de maior similaridade florística (15 e 16) ocorreram 12 espécies em comum, sendo estas Conceveiba guianensis, Duguetia chrysea, Eschweilera amazonica, E. bracteosa, Hevea guianensis, Iryanthera laevis, Licaria cannela, Mabea piriri, Pouteria filipes, Pouteria sp. 2, Protium apiculatum e $P$. trifoliolatum. Nas parcelas de menor similaridade ocorreram três espécies no total, $\operatorname{com} E$. bracteosa a única espécie comum às parcelas mais similares.

Para o coeficiente qualitativo de Sorensen, a variação foi de 28,1 a 35,8, indicando maior similaridade florística entre as parcelas 5 e 6 (Figura $5 \mathrm{c}$ ). As espécies comuns às duas parcelas foram Abuta rufescens, Brosimum rubescens, Chrysophyllum prieurii, Eschweilera odora, E. tessmannii, Licania beteromorpha, L. octandra, Mabea caudata, Ocotea cinerea, Oenocarpus bacaba, Protium apiculatum e Swartzia reticulata. Confrontando as similaridades observadas para os índices de Porcentagem e Sorensen, nota-se que Protium apiculatum foi a única a estar presente em ambos coeficientes, possivelmente relacionada a alta densidade dessa espécie na amostragem, corroborando para a sua aparição em todas as parcelas avaliadas (Figura 5).

As classificações obtidas segundo os diferentes grupos de plantas estão ilustradas nas figuras (Figura $5 \mathrm{a}$-d). Os dendogramas formados a partir dos dois índices de similaridade seguiram padrão praticamente idênticos, evidenciando tendências de formação de seis grupos de parcelas: (3 e $4 ; 2,5$ e 6 ; 8 e $9 ; 15$ e $16 ; 18-20)$. No primeiro, terceiro e penúltimo grupos, as parcelas encontram-se
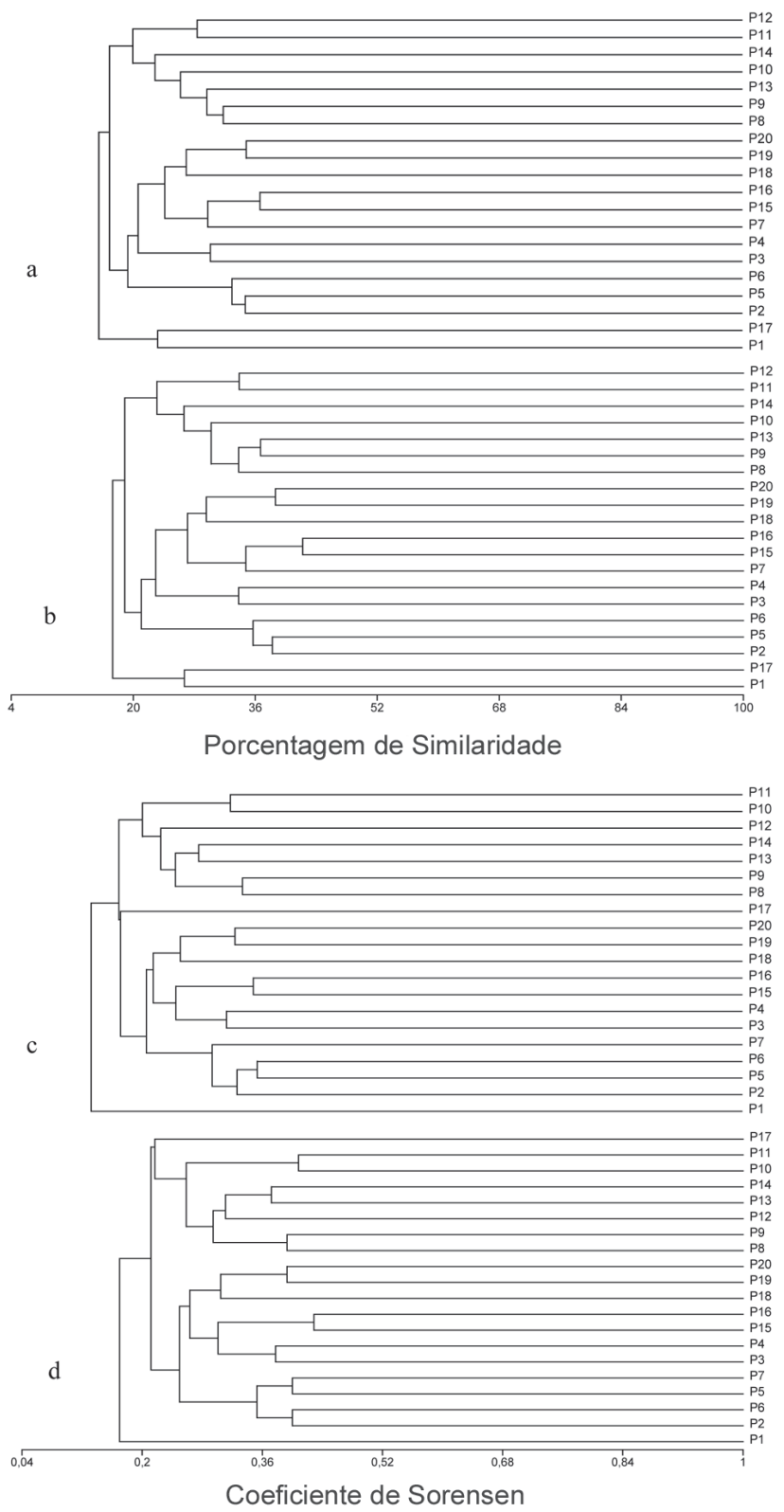

Figura 5 - Análises de agrupamento pelos índices de Porcentagem (a, b) e Sorensen (c, d) no ambiente florestal estudado. Manaus, Amazonas, Brasil. a e c $=$ todas as espécies; b e $\mathrm{d}=$ excluindo as espécies "localmente raras". Manaus, Amazonas, Brasil. 


\section{ACTA \\ AMAZONICA}

FLORÍSTICA E FITOSSOCIOLOGIA DE UMA FLORESTA

DE VERTENTE NA AMAZÔNIA CENTRAL, AMAZONAS, BRASIL próximas entre si. No segundo grupo, a ordem entre as parcelas 2, 5 e 6 não é consistente. As relações dentro dos demais grupos não ficaram bem evidentes, denotando mais uma vez a heterogeneidade desse ambiente florestal.

A máxima similaridade florística obtida a partir dos índices testados ficou em 36,2\%, sustentando outros resultados (Campbell, 1994; Oliveira, 1997; Ferreira \& Prance, 1998) que documentaram valores oscilando entre 10 a $36 \%$ para as florestas de terra firme da Amazônia. Neste estudo pôde-se verificar ainda que, quanto menor a distância geográfica entre parcelas de 500 $\mathrm{m}^{2}$, maior foi a similaridade estimada (Figura $5 \mathrm{~b}-\mathrm{d}$ ).

É importante ressaltar que a baixa similaridade anotada neste trabalho pode ser explicada, em parte, pelo alto percentual de espécies "localmente raras", presentes na amostragem (49\%). Quando estas são excluídas da análise, observa-se redução na dissimilaridade florística entre parcelas, com as similaridades máximas aumentando em 6,2 e 7,6\%, para os índices de Porcentagem e Sorensen, respectivamente (Figura 5 b, d).

\section{CONCLUSÕES}

A floresta de vertente da ZF-2 apresenta-se bem diversificada, com Sapotaceae, Lecythidaceae, Fabaceae, Caesalpiniaceae e Chrysobalanaceae compondo as cinco famílias com maior riqueza de espécies e número de indivíduos por hectare;

As espécies Eschweilera bracteosa e Protium apiculatum são as mais importantes para o equilíbrio ecológico da comunidade no ambiente florestal avaliado;

Houve predomínio do padrão aleatório de distribuição espacial das espécies no hectare amostrado;

Os índices de diversidade e uniformidade de Shannon-Wiener de 5,01 nats.indivíduo ${ }^{-1}$ e 0,91 , respectivamente, são considerados altos no contexto de estudos semelhantes na região;

A heterogeneidade edáfica e topográfica da área, as taxas de recrutamento de novos indivíduos e/ou de espécies "localmente raras" à comunidade local, podem ter contribuído para as altas dissimilaridade $(36,2 \%)$ e diversidade florísticas encontradas neste trabalho.

\section{BIBLIOGRAFIA CITADA}

Amaral, I.L. 1996. Diversidade florística em floresta de terra firme, na região do rio Urucu- AM. Tese de Mestrado, Instituto Nacional de Pesquisas da Amazônia/Universidade Federal do Amazonas, Manaus, Amazonas. 121pp.

Amaral, I.L.; Matos, F.D.A.; Lima, J. 2000. Composição florística e estrutural de um hectare de floresta densa de terra firme no Rio Uatumã, Amazônia, Brasil. Acta Amazonica, 30:377-392.

Campbell, D.D.; Daly, D.C.; Prance, G.T.; Maciel, U.N. 1986. Quantitative ecological inventory of terra firme and varzea tropical forest on the rio Xingu, Brazilian, Amazon. Brittonia, 38:369-393.

Clinebell, R.R., Phillips, O.L., Gentry, A.H.; Stark, N.; Zuuring, H. 1995. Prediction of neotropical tree and liana species richness from soil and climatic data. Biodiversity and Conservation, 4:56-90.
Cronquist A. 1981. An integrated system of classification flowering plants. Columbia University Press, New York, USA.1262 pp.

Ferreira, L.V.; Prance, G.T. 1998. Species richness and floristic composition in four hectares in the Jaú National Park in upland forests in Central Amazonia. Biodiversity and Conservation, 7: 1349-1364.

Gentry, A.H. 1988. Changes in plant community diversity and floristic composition on environmental and geographical gradients. Annals of the Missouri Botanical Garden, 75:1-34.

Higuchi, N.; Santos, J.;Vieira, G.; Ribeiro, R.J.; Sakurai, S.; Ishizuca, M.; Sakai, T.; Tanaka, N.; Saito, S. 1998. Plant structural analysis of a pristine tropical moist forest in Cuieiras river basin region, ZF - 2, Manaus - AM, Brazil. In: Higuchi, N.; Campos, M.A.A.; Sampaio, P.T.B.; Santos, J. (Eds.). Pesquisas florestais para a conservação e reabilitação de áreas degradadas da Amazônia. Instituto Nacional de Pesquisas da Amazônia, Manaus, p. 53-81.

Houghton, R. A.; Skole, D.L.; Nobre, C.A.; Hackler, J.L.; Lawrence, K.T.; Chomentowski, W.H. 2000. Annual fluxes of carbon from deforestation and regrowth in the Brazilian Amazon. Nature, 403:301-304.

Jardim, F.C.S.; Hosokawa, R.T. 1986/1987. Estrutura da floresta equatorial úmida da Estação Experimental de Silvicultura Tropical do INPA. Acta Amazonica, 16/17(único):411-508.

Jardim, F.C.S. 1995. Comportamento da regeneração natural de espécies arbóreas em diferentes intensidades de desbastes por anelamento, na região de Manaus, AM. Tese de Doutorado, Universidade Federal de Viçosa, Viçosa, Minas Gerais. 169pp.

Knight, D.H. 1975. A phytosociological analysis of species-rich tropical forest on Barro Colorado Island, Panama. Ecological Monographs, 45:259-28.

Krebs C.J. 1989. Ecological methodology. Harper \& Row, New York, USA. 654pp.

Laurance, W. F., M. A.; Cochrane, S.; Bergen, P. M.; Fearnside, P.; Delamônica, C.; Barber, S. d'Angelo, Fernandes, T. 2001. The future of the Brazilian Amazon: development trends and deforestation. Science, 291:438-439.

Lima Filho, D.A.; Matos, F.D.A.; Amaral, I.L.; Revilla, J.; Coêlho, L.S.; Ramos, J.F.; Santos, J.L. 2001. Inventário florístico de floresta ombrófila densa de terra firme, na região do Rio UrucuAmazonas, Brasil. Acta Amazonica, 31:565-579.

Magurran A.E. 1988. Ecological diversity and its measurement. Princeton University Press, New Jersey, USA, 192pp.

Matos, F.D.A.; Amaral, I.L. 1999. Análise ecológica de um hectare em floresta ombrófila densa de terra-firme, estrada da várzea, Amazonas, Brasil. Acta Amazonica, 29:365-379.

Mori, A.S.; Boom, B. 1983. Ecological importance of Myrtaceae in na eastern Brazilian wet forest. Biotropica, 15:68-70.

Müller-Dombois, D.; Ellemberg, H. 1974. Aims and methods for vegetation ecology. John Wiley \& Sons, New York, USA. 547pp.

Nobre, C. A.; Sellers, P.; Shukla, J. 1991. Amazonian deforestation and regional climate change. Journal of Climate, 4: 957-988.

Oliveira, A.A.; Mori, S.A. 1999. A central Amazonian terra firme forest. I. High tree species richness on poor soils. Biodiversity and Conservation, 8:1219-1244. 


\section{ACTA AMAZONICA}

FLORÍSTICA E FITOSSOCIOLOGIA DE UMA FLORESTA

DE VERTENTE NA AMAZÔNIA CENTRAL, AMAZONAS, BRASIL
Oliveira, A.N.; Amaral, I.L.; Nobre, A.D.; Couto, L.B.; Sado, R.M. 2003. Composition and floristic diversity in one hectare of a upland forest dense in Central Amazonia, Amazonas, Brazil. Biodiversity and Conservation (in press).

Phillips, O.L.; Hall, P.; Gentry, A.H.; Sawyer, S.A.; Vásquez, R. 1994. Dynamics and species richness of tropical rain forests. Proceedings of the Naional Academy of Sciences of the USA, 91:2805-2809

Porto, M.L.; Longhi, H.M.; Citadini, V.; Ramos, R.F.; Mariath, J.E.A. 1976. Levantamento fitossociológico em área de "mata-debaixio", na estação Experimental de Silvicultura Tropical - INPA - Manaus - Amazonas. Acta Amazonica, 6:301-318.

Prance, G.T.; Rodrigues, W.A.; Silva, M.F. 1976. Inventário florestal de um hectare de mata de terra firme, $\mathrm{km} 30$ da estrada ManausItacoatiara. Acta Amazonica, 6:9-35.

RADAM, 1978. Programa de Integração Nacional. Levantamentos de Recursos Naturais. v.18 (Manaus) - Radam (projeto) DNPM, Ministério das Minas e Energia. Brasil. 626pp.

Rabelo, F.G.; Zarin, D.J.; Oliveira, F.A.; Jardim, F.C.S. 2002. Diversidade, composição florística e distribuição diamétrica do povoamento com DAP $\geqslant 5 \mathrm{~cm}$ em região de estuário no Amapá. Revista de Ciências Agrárias, 37:91-112.

Ranzani, G. 1980. Identificação e caracterização de alguns solos da Estação Experimental de Silvicultura Tropical do INPA. Acta Amazonica, 6:7-41.

Ribeiro, J.E.L.S, Hopkins, M.J.G.; Vicentini, A.; Sothers, C.A.; Costa, M.A.S.; Brito, J.M.; Souza, M.A.D.; Martins, L.H.P.; Lohmann, L.G.; Assunção, P.A.C.L.; Pereira, E.C.; Silva, C.F.; Mesquita, M.R.; Procópio, L.C. 1999. Flora da Reserva Ducke. Guia de Identificação das Plantas Vasculares de uma Floresta de Terra-firme na Amazônia Central, Instituto Nacional de Pesquisas da Amazônia, Manaus, 793pp.

Rossi, L.M.B.; Higuchi, N. 1998. Comparação entre métodos de análise de padrão espacial de oito espécies arbóreas de uma floresta tropical úmida. In: Gascon, C.; Moutinho, P. (Eds). Floresta Amazônica: Dinâmica, Regeneração e Manejo. Instituto Nacional de Pesquisas da Amazônia, Manaus, Amazonas. p. 41-60.
Santana, C.A.A. 2002. Estrutura florística de fragmentos de florestas secundárias de encosta no Município do Rio de Janeiro. Tese de Mestrado, Universidade Rural do Rio de Janeiro, Seropédica, Rio de Janeiro. 133pp.

Skole, D.;Tucker, C. 1993. Tropical deforestation and habitat fragmentation in the Amazon: satellite data from 1978 to 1988. Science 260:1905-1910

Steege, H.; Sabatier, D.; Castellanos, H.; Andel, T.V.; Duivenvoorden, J.; Oliveira, A.A.; Renske, E.; Lilwah, R.; Maas, P.; Mori, S. 2000. A regional perspective: Analysis of Amazonian floristic composition and diversity that includes a Guyana Shield. In: Steege, H. (Ed.). Plant Diversity in Guyana: Whit recommendations for a National Protected Areas Strategy. The Tropenbos Foundation, Wageningen, p. 19-32.

Tello, J.C.R. 1995. Aspectos fitossociológicos das comunidades vegetais de uma toposseqüência da Reserva Florestal Ducke do INPA. Tese de Doutorado, Instituto Nacional de Pesquisas da Amazônia/Universidade Federal do Amazonas, Manaus, Amazonas. 335pp.

Uhl, C.; Murphy, P.G. 1981. Composition, Structure and Regeneration of a tierra firme Forest in the Amazonian Basin of Venezuela. Tropical Ecology, 22: 219-237.

RECEBIDO EM 07/07/2003

ACEITO EM 21/10/2003 